\title{
Traduzione e rispetto filologico: alcune osservazioni sulla traduzione di alcune poesie della scuola siciliana al Labestrad della UFF
}

\author{
Guido Alberto Bonomini ${ }^{1}$ \\ Universidade Federal Fluminense \\ wildoalberto@yahoo.com.br
}

RIASSUNTO: La traduzione che abbia uno scopo didattico deve sempre rispettare la voce dell' autore, quindi non è possibile ricreare il testo conmere finalità artistiche. L'esperienza della traduzione di alcune poesie della scuola siciliana ci ha costretto ad avvicinarci al testo e aggiungere delle note per spiegare dei termini topici.

Parole-chiave: Traduzione. Rispetto all'originale. Didattica filologicamente corretta.

ABSTRACT: Translation with a didacticscope must always respect the source text and the author's project. It is not possible to recreate a text only in its artistic purpose. The translation experience of working with some poems from the Sicilian School brought us really close to the source text and made us add notes so as to further explain specific terms from the original context.

Keywords: Translation. Fidelity. Philologically correct didactics.

\section{Essere traduttore}

È spesso ricorrente leggere un adagio che suona più o meno così: è difficile il compito del traduttore.

Dipende, risponderei io, ossia, è evidente che non è un'operazione quella del tradurre alla portata di qualsiasi persona che si avvicini a una lingua diversa dalla sua. Si rende quindi necessario che un futuro traduttore conosca adeguatamente la lingua straniera come la sua e che non tenti di arrampicarsi sugli specchi di fronte alle immancabili difficoltà che gli si presentino, tentando interpretazioni cosiddette artistiche, ma in realtàad sensum, risultati derivanti soprattutto dalla scarsa conoscenza anche della lingua di partenza.

${ }^{1}$ Dottore in Letteratura Comparata (UFF), ha il Master in Letteratura italiana presso la UFRJ. Attualmente, è docente di Lingua, Letteratura e Cultura italiane presso la UFF. 
Non esiste, di fatto, una traduzione che malgrado letterale, sia mot a mot, la lingua sia di partenza che di arrivo, deve essere considerata sempre in un contesto. Considerare il contesto è oltremodo fondamentale non solo a livello linguistico, ma anche e soprattutto storico e culturale. Sappiamo tuttavia che, nel passaggio da una lingua a un'altra, si opera sempre una sorta di ricreazione, il testo di arrivo non sarà mai uguale in tutto e per tutto al testo di partenza. Come anche ricordava I. Calvino nel suo libro, Testo scritto e Testo non scritto, due lingue portano in sé due modi diversi di sentire le parole; a questo proposito, come è diversa, ad esempio, l'idea di pane in italiano e l'equivalente pãonel portoghese del Brasile. Per un italiano il pane accompagna tutti i piattidalla colazione al pranzo alla cena, tanto che l'idea evocata dalla parola pane abbraccia nella cultura italiana una visione pressoché onnipresente sulla sua tavola; per un brasiliano invece parlare di pane, evoca il breakfast, ossia il caféda manbã o al massimo uno spuntino pomeridiano. E questa è solo una parola isolata in un universo culturale della lingua!

Poi non possiamo non contemplare il contesto storico, come nel caso della traduzione di alcune poesie della cosiddetta scuola siciliana, che risalgono alla prima metà del XIII secolo. In questo caso è necessaria una prima traduzione, dalla lingua antica alla moderna, che il traduttore deve fare per comprendere per primo lui il testo, altrimenti sarà inutile tradurre ciò che neanche il traduttore comprende. Poi tentare di avvicinarsi al testo di partenza, sia linguisticamente, sia anche cronologicamente, usando una lingua non eccessivamente moderna nella lingua di arrivo.

Quanto alla lingua della poesia siciliana, in particolare, bisogna considerare che è stata una creazione artificiale ed eminentemente poeticoletteraria che parte da un contesto fonico dialettale, il siciliano colto appunto, arricchito di prestiti e calchi semantici provenienti da un contesto letterario franco-provenzale, funzionale e fruibile per lo più a quanti erano interni alla 
corte di Federico II o che, quantomeno, intendessero di poesia d'amore. Credo che sia necessaria una premessa, quando ci si avvicina e si tenta di tradurre testi letterari e oltretutto poetici delle origini della letteratura italiana: la lingua usata per la produzione poetica, non è una lingua d' uso, ma solo di ambito poetico, così come, in toto la lingua italiana, è una lingua letteraria, ossia nasce da una esercitazione, oltremodo artificiale che gli scrittori operarono a partire da una tradizione composita e soprattutto scritta. Insomma, nel caso della lingua usata dai poeti della corte federiciana, dobbiamo immaginare che questa non fossedirettamente e unicamente ispirata alla lingua popolare, ovviamente (nel senso che questa lingua artificiale, non coincideva con la lingua d'uso dei contadini e pescatori siciliani del XIII secolo), ma che questa capta a livello artistico solo alcuni suoni e alcune forme della lingua dialettale e viene poi arricchitada una veste puramente letteraria. Questo, per me, è il contesto sul quale anche un traduttore deve ragionare. E' necessario quindi in primo luogo capire cosa significa il testo nell'epoca, per l'epoca in cui è stata scritta, per poi inserirlo in un contesto linguistico della lingua di arrivo, quando si traduce, senza alterare i contenuti posti dall'autore.

Qualsiasi ricreazione, anche se si tratta di una lingua poetica, non è a mio avviso permessa, la traduzione deve, comunque, tenere conto, così come la filologia, delle intenzioni dell'autore, il traduttore mai dovrà essere l'autore. E invece si vedono moltissime traduzioni, o quantomeno definite tali, di testi poetici italiani che non sono altro che delle chiare rielaborazioni che si allontanano anni luce dalla voce dell'autore, che si basano sulla giustificazione che si debba mantenere la sonorità del verso, a costo anche di pervertire il senso generale del testo di origine. A mio avviso queste opere dovrebbero essere denominate così libera interpretazione a partire dall'opera X. La traduzione di un testo poetico può, penso, esser riprodotta anche in prosa, ma 
Traduzione e rispetto filologico: alcune osservazioni sulla traduzione

di alcune poesie della scuola siciliana al Labestrad della UFF

Guido Alberto Bonomini

con testo a fronte. La finalità, come anche questa proposta di traduzione ${ }^{2}$, per la rivista Ecos de Linguagem, di alcuni componimenti dei poeti della scuola siciliana ${ }^{3}$, è quella di poter avvicinare il lettore al testo antico, non allontanarlo o confonderlo, creando un testo che poco o nulla ha a che vedere col testo di partenza, facendo poi credere all'incauto lettore che si tratti di una poesia di Stefano Protonotaro.

\section{Pirmeu cori alligrari, di Stefano Protonotaro, alcune difficoltà di adattamento alla lingua portoghese}

Nella traduzione del componimento di Stefano Protonotaro, Pirmeu cori alligrari, che è una delle poche se non l'unica ad essere arrivata a noi in veste siciliana e non toscanizzata, una delle difficoltà che si è presentata, subito nella prima parte della poesia è la traduzione di un termine funzionale nella poesia d'amore provenzale e preso quindi in prestito dalla poesia siciliana: joi.

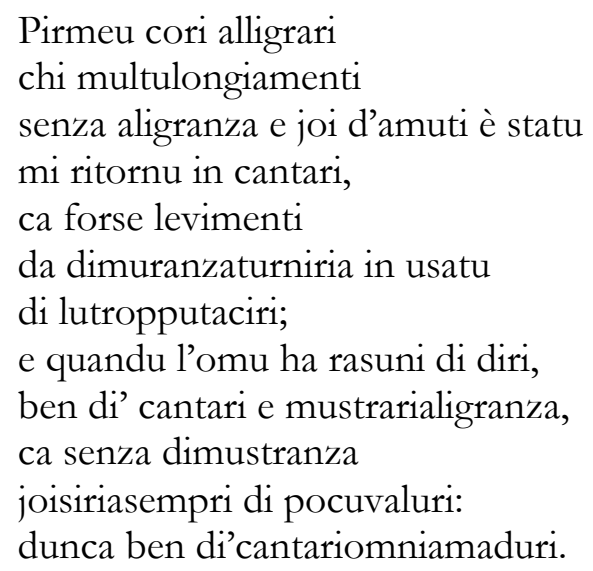

Ora, passiamo alla versione in italiano, per chiarire alcuni punti che anche il traduttore deve comprendere per poter poi passare nella lingua d'arrivo, nel caso specifico, il portoghese del Brasile:

Per rallegrare il mio cuore,

\footnotetext{
2 Un lavoro congiunto fra il LABESTRAD (UFF) e la UERJ.
}

${ }^{3}$ Dalla collana, di Roberto Antonelli, I poeti della scuola siciliana. 
Traduzione e rispetto filologico: alcune osservazioni sulla traduzione

di alcune poesie della scuola siciliana al Labestrad della UFF

Guido Alberto Bonomini

che è stato molto a lungo senza

allegria e gioia d'amor,

torno a cantare, poiché forse per il

troppo tacere potrei facilmente

prendere questa abitudine (direstare in silenzio);

e quando uno ha motivo di parlare,

devecerto cantare e mostrare allegria,

in quanto la gioia

sarebbe sempre di poco valore

se non la si dimostrasse:

dunque ogni amante deve cantare.

Ora una traduzione che ci porti verso il contenuto della poesia ma che non riproduce le rime:



La poesia tradotta ha uma mera finalità didattica, ossia avvicina il lettore o lo studente straniero all'universo della poesia d'amore delle origini ed è fruibile e utile se fornita con testo a fronte.

Ora, come introducevo, la forzatura è nella traduzione di joi, con joia, che viene comunque accompagnata da nota, spiegando che joi deriva dal latino gaudium e anche in italiano, così come in portoghese è un caso di contaminazione linguistica, ossia non arriva per via diretta dal latino alle due lingue romanze in questione, nel caso l'italiano e il portoghese, ma vi arriva solo per un tramite franco-provenzale, anche a livello di contenuto. La joi nella poesia d'amore sia franco-provenzale sia quindi, come prestito, nel siciliano, 
sta a significare la gioia che produce il saper amare fedelmente una donna anche senza avere nulla in cambio. E' una parola altamente produttiva nel campo semantico amoroso, visto che ci potrebbe anche trarre in inganno, perché sottilmente potrebbe introdurre la gioia d'amore, nel caso sessuale, anche se di fatto non per tutti gli scrittori, anche provenzali è così, malgrado la forza etimologica di gaudium. Pertanto abbiamo una certa divaricazione nel significato di joi, anche tra poeti come Guglielmo IX di Aquitania, per il quale la joi può anche rappresentare piacere fisico e ad esempio in Jaufré Raudel o Bernard de Ventadorn, per i quali invece la joi è un piacere perlopiù derivante dal vassallaggio in amore. Con questo secondo senso, più puro e distante, passa nella lirica siciliana, dove mai il fatto sessuale è consumato. Esistono ovviamente delle eccezioni, come nel caso di Giacomino Pugliese in Risplendiente stella d'arbur, dove invece abbiamo in sottofondo un incontro galante. Ma nel caso specifico della traduzione di questo componimento di Stefano Protonotaro, come degli altri componimenti e autori tradizionalmente iscritti tra quelli della scuola federiciana, $j o i$ non ha mai un connotato sessuale.

Ora, nel portoghese, anche in quello del Brasile, malgrado esista la parola in una fraseologia del tipo: "tudo joia?", "essa moça é uma joia!", dove quindi è implicito che joia ha valore di bello, piacevole, (sinonimo di: beleza), traducibile con tutto bene, bello, divertente, e, nonostante i dizionari ${ }^{4}$ in uso riportino sia il senso originale del termine sia i significati legati alla lingua d'uso, joia (ossia monile prezioso, anche in senso morale), per quanto riguarda la conoscenza linguistica dei lettori, rimane una parola distante dai significati legati all' etimologia franco-provenzale

Pertanto, nel valore che ha questa espressione idiomatica (tudo joia!)ci si avvicina oltremodo al valore di joi da gaudium. Nel caso del portoghese gozo deriva da gaudium, ma in questo caso non sarebbe appropriato perché

\footnotetext{
${ }^{4}$ Cfr. Dicionário Aurélio e Houaiss.
} 
Traduzione e rispetto filologico: alcune osservazioni sulla traduzione

di alcune poesie della scuola siciliana al Labestrad della UFF

Guido Alberto Bonomini

sinonimo di raggiungimento di piacere sessuale. A mio avviso in casi come questi dove esiste, anche se solo a livello colto e letterario l'uso di una parola, credo sia necessario mantenere la parola, spiegandone anche il cammino etimologico e ovviamente il valore topico che ha nella poesia d'amore delle origini dalla Provenza alla Sicilia e dalla Sicilia alla Toscana. La nota per joia, derivato da joi, ossia felicità nel pensiero d'amore, è d'obbligo, visto che nella lingua portoghese corrente non è direttamente comprensibile.

Umberto Eco, in Dire quasi la stessa cosa, afferma che a volte, però, è opportuno mettere in risalto gli aspetti fonico-prosodici, quando questa é direttamente l'intenzione dell'autore. Cita a questo proposito varie delle sue traduzioni dal francese e dall'inglese dove il semiologo afferma che spesso ha dovuto rinunciare alla reversibilità lessicale e sintattica.

In conclusione, varie volte nel corso della mia traduzione di questi brani ho rinunciato a una reversibilità lessicale e sintattica perché ritenevo che il livello veramente pertinente fosse quello metrico e su quello ho giocato. Quindi non mi preoccupavo tanto della reversibilità letterale, quanto di provocare lo stesso effetto che, secondo la mia interpretazione, il testo voleva provocare nel lettore (ECO, 2010, p. 7879).

A partire da una posizione del genere, Paulo Rónai (RÓNAI, 2012, p. 14), nel suo libro Escola de tradutores, vede una pressoché impossibilità teorica di una traduzione letteraria, implicando che la traduzione è arte. Nel libro Metalinguagens e outras metas, di Haroldo de Campos, viene citato Ronai:

O objetivo de toda arte não é algo impossível? O poeta exprime (o que quer exprimir) o inexprimível, o pintor reproduz o improduzível, o estatuário fixa o infixável. Não é surpreendente, pois, que o tradutor se empenhe em traduzir o intraduzível (RÓNAI, 2012, p. 14 apud CAMPOS, 2006, p. 34-35).

Questa posizione di Rónai la vedo estrema, non sempre il traduttore traduce l'intraducibile. Bisogna a volte optare, come insegna I. Calvino a scegliere un solo aspetto nella traduzione, come l'aspetto letterale o quello ritmico, implicando così una rinuncia al resto. Ossia questa posizione 
Traduzione e rispetto filologico: alcune osservazioni sulla traduzione

di alcune poesie della scuola siciliana al Labestrad della UFF

Guido Alberto Bonomini

definibile, artistica, di libera creazione, credo sia oltremodo ambigua e mal interpretabile, nel senso che molti traduttori si possano sentire autorizzati a far scempio del testo dell'autore, trasformandolo radicalmente. Giochi di parole, d'accordo, possono esser riprodotti con giochi di parole, ritmici, anche nella lingua di arrivo, ma è opportuno fare una premessa, dichiarando che la traduzione è liberamente tratta dall'originale e riproduce suoni ma non contenuti. La posizione di Haroldo de Campos è ancora più estrema, apre definitivamente la strada a un processo ricreativo del testo con la finalità di mantenere un parallelismo tra il valore estetico del testo di partenza, preservando così sonorità e ritmi nel testo di arrivo,

(..) num produto que só deixe de ser fiel ao significado textual para ser inventivo, e que seja inventivo na medida mesma em que troncando deliberadamente, a fidelidade aosignificado para conquistar uma lealdade maior ao espirito do original translado ao próprio signo estético visto como entidade total, indivisa, na sua realidade material (no seusuportefísico, quemuitasvezes deve tomar a dianteiranaspreocupações do tradutor) e na sua cargaconceitual (CAMPOS, 2006, p. 47).

Definitivamente, non sono affatto d'accordo con questa posizione di Haroldo de campos, mai e poi mai, mi pare, si deveprivilegiareil piano estetico-fonico-ritmico a discapito di quello del contenuto. Che utilità pratica avrà un testo completamente trasformato in un'altra lingua, parlando non di quasi la stessa cosa ma di qualcosa completamente differente?

\section{Riferimenti Bibliografici}

ECO, Umberto. Dire quasi la stessa cosa. Milão: Bompiani, 2010.

RÓNAI, Paulo. Escola de tradutores. Rio de Janeiro: José Olympio, 2012.

CAMPOS, Haroldo de. Metalinguagem e outras metas. São Paulo: Perspectiva, 2006. 


\section{Tradução e respeito filológico: Algumas observações sobre a tradução de poesias da escola siciliana para o Labestrad da} $\mathrm{UFF}^{5}$

RESUMO: A tradução com escopo didático deve sempre respeitar o texto de partida, a proposta do autor. Não é possível recriar o texto apenas com finalitdade artística. A experiência da tradução de algumas poesias da Escola Siciliana levou-nos à extrema aproximamação do texto de partida e a acrescentar notas explicativas para elucidar termos específicos do contexto original.

Palavras-chave: Tradução. Fidelidade. Didática filologicamente correta.

ABSTRACT: Translation with a didacticscope must always respect the source text and the author's project. It is not possible to recreate a text only in its artistic purpose. The translation experience of working with some poems from the Sicilian School brought us really close to the source text and made us add notes so as to further explain specific terms from the original context.

Keywords: Translation. Fidelity. Philologically correct didactics.

\section{Ser tradutor}

É recorrente ouvirmos um ditado que soa mais ou menos assim: "É difícil a tarefa do tradutor".

\footnotetext{
${ }^{5}$ Tradução de Cecilia Maculan Adun, dotoranda em Semiótica na UFF.

${ }^{6}$ Doutor em Literatura Comparada (UFF), é Mestre em Literatura Italiana (UFRJ). Atualmente, é Professor de Língua e Literatura Italiana na UFF.
} 
Traduzione e rispetto filologico: alcune osservazioni sulla traduzione di alcune poesie della scuola siciliana al Labestrad della UFF

Guido Alberto Bonomini

Depende, eu responderia, ou seja, é evidente que a operação do tradutor não é factível a qualquer pessoa que se aproxime de uma língua diferente da sua. Torna-se necessário, portanto, que um futuro tradutor conheça adequadamente tanto a língua estrangeira como a sua e que não se desgaste em vão diante das inevitáveis dificuldades que se lhe apresentem, buscando interpretações ditas artísticas, mas, na verdade, derivadas, sobretudo, do escasso conhecimento da língua de partida. Não existe, de fato, uma tradição que malgrado literal, seja mot a mot. A língua, tanto de partida quanto de chegada, deve ser considerada sempre em um contexto. Considerar o contexto é extremamente fundamental não apenas no nível linguístico, mas também, e, sobretudo, no nível histórico e cultural. Sabemos, todavia, que na passagem de uma língua para outra, opera-se sempre uma espécie de recriação: o texto de chegada não será jamais igual ao texto de partida. Como nos lembra Italo Calvino, em seu livro Testo scritto e testo non scritto, duas línguas trazem em si dois modos diversos de sentir as palavras; a esse propósito, como é diferente, por exemplo, a ideia de pane (pão) em italiano e o equivalente pão no português do Brasil. Para um italiano, o pane acompanha todos os pratos, desde o café da manhã ao almoço e ao jantar, tanto que a ideia evocada pela palavra pane abarca, na cultura italiana, uma visão quase onipresente sobre sua mesa. Para um brasileiro, por outro lado, falar de pão, evoca, no máximo, o café da manhã, ou, talvez um lanche da tarde. E essa é apenas uma palavra isolada em meio a um universo cultural da língua!

Não podemos não contemplar o contexto histórico, especialmente no caso da tradução de poesias da assim dita escola siciliana, as quais datam da primeira metade do século XIII. Nesse caso é necessária uma primeira tradução, da língua antiga para a moderna, que o tradutor deve executar para que, primeiramente, possa ele próprio compreender o texto original, pois, do contrário, será inútil traduzir algo que nem mesmo o tradutor compreende. A 
seguir, deve tentar se aproximar do texto de partida, seja linguisticamente, seja cronologicamente, usando, no idioma de chegada, uma língua não excessivamente moderna. No que concerne à língua da poesia siciliana, em particular, é necessário levar em consideração que se trata de uma criação artificial e eminentemente poético-literária que parte de um contexto fonético dialetal, o siciliano culto, enriquecido por empréstimos e calcos semânticos provenientes de um contexto literário franco-provençal funcional e desfrutável em geral por aqueles que pertenciam à corte de Federico II ou que, ao menos, entendessem um pouco de poesia de amor. Creio que seja necessária uma premissa quando um tradutor se aproxima de textos literários e tenta traduzi-los, especialmente textos poéticos das origens da literatura italiana: a língua usada para a produção poética não é uma língua de uso, mas apenas de âmbito poético, assim como, de modo geral, a língua italiana é uma língua literária, ou seja, uma língua que nasce de uma exercitação, exageradamente artificial, que os escritores realizaram a partir de uma tradição composta e, sobretudo, escrita. Em suma, no caso da língua usada pelos poetas da corte de Federico, devemos imaginar que essa não fosse direta e unicamente inspirada na língua popular, obviamente, (no sentido de que essa língua artificial não coincidia com a língua de uso dos camponeses e pescadores sicilianos do século XIII), mas tal língua, no nível artístico, capta apenas alguns sons e algumas formas do dialeto falado, sendo então enriquecida por uma veste puramente literária. Esse, a meu ver, é o contexto sobre o qual também um tradutor deve raciocinar. É necessário, pois, em primeiro lugar, entender o que significa o texto na e para a época em que foi escrito, para então inseri-lo no contexto linguístico da língua de chegada, quando, então, se traduz sem que haja alteração dos conteúdos colocados pelo autor. 
Traduzione e rispetto filologico: alcune osservazioni sulla traduzione di alcune poesie della scuola siciliana al Labestrad della UFF

Guido Alberto Bonomini

Toda e qualquer recriação, ainda que se trate de uma língua poética, não é, em minha visão, permitida. A tradução deve, em todo caso, considerar, assim como o faz a filologia, as intenções do autor. $\mathrm{O}$ tradutor não deverá jamais ser o autor. E, ao contrário, veem-se muitas traduções, ou pelo menos definidas como tais, de textos poéticos que não são mais do que claras reelaborações que se distanciam anos-luz da voz do autor e que se baseiam na justificativa de que se deva manter a sonoridade do verso, sob pena inclusive de perverter o sentido geral do texto original. Penso que obras como essas deveriam ser denominadas livres interpretações a partir da obra original X. A tradução de um texto poético pode, em minha visão, ser reproduzida também em prosa, mas com o original a fronte. A finalidade, como também a proposta de tradução, para a revista Eco da Linguagem, de algumas composições de poetas da escola siciliana, é aquela de poder realmente aproximar o leitor do texto antigo, sem afastá-lo ou confundi-lo com um texto que pouco ou nada tem em comum com o texto de partida, fazendo, pois, com que o incauto leitor acredite estar diante de uma poesia de, por exemplo, Stefano Protonotaro.

\section{Pir meu cori alligrari, de Stefano Protonotaro: algumas dificuldades de adequação para a língua portuguesa}

$\mathrm{Na}$ tradução da composição de Stefano Protonotaro, Pir meu cori alligrari, que é uma das poucas, senão a única, que chegou até nós em veste siciliana e não em forma toscana, uma das dificuldades que se apresenta, logo na primeira parte da poesia, é a tradução de um termo funcional na poesia de amor provençal e emprestado à poesia siciliana: joi.

Pir meu cori alligrari chi multu longiamente senza aligranza e joi d'amuti è statu mi ritornu in cantari, ca forse levimenti 
Traduzione e rispetto filologico: alcune osservazioni sulla traduzione

di alcune poesie della scuola siciliana al Labestrad della UFF

Guido Alberto Bonomini

da dimuranza turniria in usatu

di lu troppu taciri;

e quandu l'omu ha rasuni di diri, ben di' cantari e mustrari aligranza, joi siria sempri di pocu valuri:

dunca ben di' cantar omni amaduri.

Passemos, agora, à versão em italiano, para esclarecer alguns pontos que também o tradutor deve compreender para poder atingir a língua de chegada, no caso, o português do Brasil:

Para rallegrare il mio cuore, che è stato molto a lungo senza allegria e gioia d'amore, torno a cantar, poiché forse per il troppo tacere potrei prendere questa abitudine (di restare in silenzio); e quando uno ha motivo di parlare, deve certo cantare mostrare allegria, in quanto la gioia sarebbe sempre di poco valore se non la dimostrasse: dunque ogni amante deve cantare.

Agora, uma tradução que nos leve até o conteúdo da poesia, mas que não reproduz, necessariamente, suas rimas:

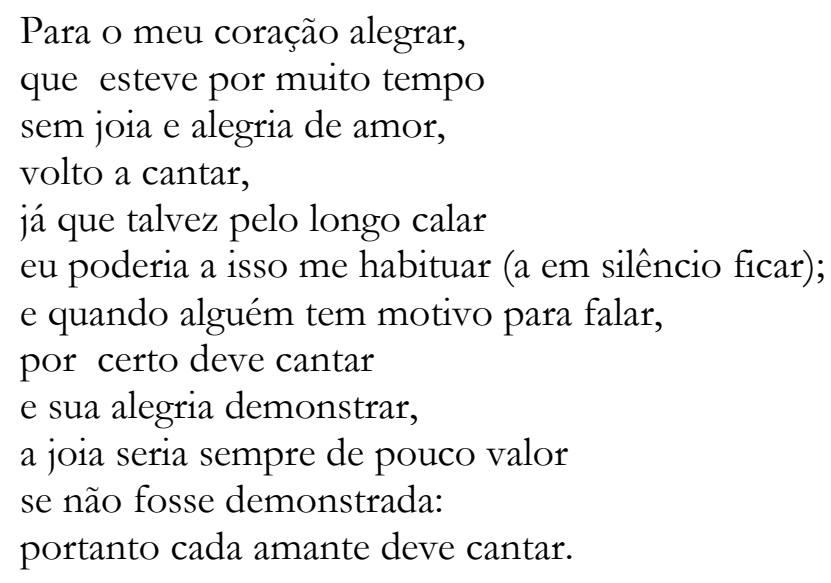

A poesia traduzida tem uma mera finalidade didática, a saber, aproxima o leitor e o estudante estrangeiro do universo da poesia de amor das origens e é deleitável e útil caso seja apresentada juntamente ao texto original. 
Traduzione e rispetto filologico: alcune osservazioni sulla traduzione di alcune poesie della scuola siciliana al Labestrad della UFF

Guido Alberto Bonomini

Como eu havia afirmado, o forçar está na tradução de joi por joia, que vem acompanhada de nota de rodapé, explicando que joi deriva do termo latino gandium e que, também em italiano, tal qual ocorre no português, tratase de um caso de contaminação linguística. Em outras palavras, a chegada às duas línguas neolatinas em questão (português e italiano) não se dá por via direta a partir do latim, mas sim através da língua franco-provençal, inclusive no nível do conteúdo. A joi, na poesia de amor, tanto franco-provençal quanto siciliana, está significando a alegria que produz o saber amar fielmente uma mulher sem ter nada em retribuição. É uma palavra altamente produtiva no campo semântico amoroso que poderia nos levar ao engano, já que, sutilmente, poderia introduzir o sentido de "gioia d'amore", de conotação sexual, mesmo que isso, de fato, não se dê para todos os escritores, incluídos aí os provençais e malgrado a força etimológica de gaudium. Teremos, pois, um certo alongamento no significado de joi, mesmo para poetas como Guglielmo IX di Aquitania, para o qual a joi pode representar inclusive o prazer físico, ou, por exemplo, um Jaufré Raudel ou um Bernard de Ventadorn, para os quais a joi é um prazer predominantemente derivado da vassalagem no amor. É com esse segundo sentido, mais puro e distante, que joi entra na lírica siciliana, onde jamais o ato sexual é consumado. Existem, obviamente, exceções, como no caso de Giacomio Pugliese, em Resplendiente stella d'albur, onde, ao contrário, temos, no pano de fundo, um encontro galante. Mas no caso específico da tradução dessa composição de Stefano Protonotaro, assim como das outras composições de autores tradicionalmente inscritos na scuola federiciana, joi não tem jamais uma conotação sexual.

No português, mesmo o do Brasil, malgrado exista a palavra joi em uma fraseologia do tipo "tudo joia?" ou "essa moça é uma joia!", onde está implícito que joia tem um valor de belo, de agradável, e pode ser traduzida para italiano por "bello", "divertente", e não obstante os dicionários em uso 
Traduzione e rispetto filologico: alcune osservazioni sulla traduzione di alcune poesie della scuola siciliana al Labestrad della UFF

Guido Alberto Bonomini

reportem tanto o sentido original do termo quanto os significados ligados à língua de uso, onde joia tem o sentido de item/objeto precioso, também em sentido moral, no que concerne ao conhecimento linguístico dos leitores, permanece sendo uma palavra distante dos significados ligados à etimologia franco-provençal.

A partir, portanto, do valor que tem essa expressão, podem-se aproximar exageradamente joi (de gaudium). No caso do português, gozo deriva de gaudium, mas no caso de nossa tradução não seria apropriado, por se tratar de um sinônimo de orgasmo. Em casos como esses, onde existe, ainda que apenas num nível culto e literário, o uso efetivo de uma palavra, creio que seja necessário manter tal palavra, explicando seu caminho etimológico e o valor tópico que possui na poesia de amor das origens, da Provance à Sicília e da Sicilia à Toscana. A nota de rodapé para joia, derivada de joi, ou seja, do conceito de felicidade no pensamento de amor, é, pois, obrigatória, já que, na língua corrente de chegada, essa acepção não é imediatamente perceptível.

Umberto Eco, em Dire quase La stessa cosa, afirma que, às vezes, porém, é oportuno colocar em relevo os aspectos fônicos e prosódicos, quando essa é diretamente a intenção do autor. A esse propósito, o semiólogo cita várias de suas traduções do francês ou do inglês nas quais, frequentemente, teve de renunciar à reversibilidade lexical e sintática.

Em conclusão, muitas vezes, no decorrer de minha tradução desses
trechos, renunciei a uma reversibilidade lexical e sintática porque
percebia que o nível realmente pertinente era aquele métrico e sobre o
qual atuei. Portanto, não me preocupava tanto com a reversibilidade
literal, quanto em provocar o mesmo efeito que, segundo minha
interpretação, o texto provocava no leitor (ECO, 2010, p. 78-79).

Paulo Rónai, em seu livro Escola de tradutores, vê a impossibilidade teórica de uma tradução literária, implicando assim a definição de tradução como arte. No livro Metalinguagens e outras metas, de Haroldo de Campos, Rónai é citado: 
Traduzione e rispetto filologico: alcune osservazioni sulla traduzione

di alcune poesie della scuola siciliana al Labestrad della UFF

Guido Alberto Bonomini

O objetivo de toda arte não é algo impossível? O poeta exprime (o que quer exprimir) o inexprimível, o pintor reproduz o improduzível, o estatuário fixa o infixável. Não é surpreendente, pois, que o tradutor se empenhe em traduzir o intraduzível (RÓNAI, 2012, p. 14 apud CAMPOS, 2006, p. 34-35).

Vejo essa posição de Rónai extrema. Nem sempre o tradutor traduz o intraduzível. Penso, sim, que seja necessário, por vezes, e como ensina Italo Calvino, escolher apenas um aspecto da tradução, como, por exemplo, o aspecto literal ou o aspecto rítmico, implicando tal escolha uma renúncia aos outros aspectos. Penso que essa posição definida como artística, de livre criação, seja excessivamente ambígua e propiciadora de uma interpretação equivocada, no sentido de que muitos tradutores possam se sentir autorizados a desmembrar o texto original, transformando-o radicalmente. Jogos de palavras, concordo, podem ser reproduzidos através de jogos de palavras na língua de chegada, mas é oportuno fazer uma ressalva, declarando que se trata (nesses casos) de uma tradução trazida livremente do original, reproduzindo seus sons, mas não necessariamente seus conteúdos.

A posição de Haroldo de Campos é ainda mais extrema e abre definitivamente a estrada para um processo de recriação do texto, com a finalidade de manter um paralelismo entre valor estético do texto de partida e a sonoridade e o ritmo no texto de chegada, pensando

\footnotetext{
(...) num produto que só deixe de ser fiel ao significado textual para ser inventivo, e que seja inventivo na medida mesma em que transcenda, deliberadamente, a fidelidade ao significado para conquistar uma lealdade maior ao espírito do original transladado, ao próprio signo estético visto como entidade total, indivisa, na sua realidade material (no seu suporte físico, que muitas vezes deve tomar a dianteira nas preocupações do tradutor) e na sua carga conceitual (CAMPOS, 2006, p. 47).
}

Definitivamente, não estou de acordo com tal posicionamento defendido por Haroldo de Campos. Em nenhum caso, penso, deva-se privilegiar o plano estético-fônico-rítmico em detrimento do conteúdo apresentado pelo texto. Que utilidade, ademais, terá um texto completamente 
Traduzione e rispetto filologico: alcune osservazioni sulla traduzione

di alcune poesie della scuola siciliana al Labestrad della UFF

Guido Alberto Bonomini

transformado no momento de chegada em outra língua e falando não de quase a mesma coisa, mas de algo completamente diferente?

\section{Referências Bibliográficas}

ECO, Umberto. Dire quase la stessa cosa. Milão: Bompiani, 2010.

RÓNAI, Paulo. Escola de tradutores. Rio de Janeiro: José Olympio, 2012.

CAMPOS, Haroldo de. Metalinguagem e outras metas. São Paulo: Perspectiva, 2006. 